\title{
Rationale, design and population baseline characteristics of the PERFORM Vascular Project: an ancillary study of the Prevention of cerebrovascular and cardiovascular Events of ischemic origin with teRutroban in patients with a history oF ischemic strOke or tRansient ischeMic attack (PERFORM) trial
}

\author{
Michael G. Hennerici • Michiel L. Bots • Ian Ford • \\ Stéphane Laurent • Pierre Jean Touboul \\ Published online: 20 May 2010 \\ (C) The Author(s) 2010. This article is published with open access at Springerlink.com
}

\begin{abstract}
Purpose PERFORM is exploring the efficacy of terutroban versus aspirin for secondary prevention in patients with a history of ischemic stroke or transient ischemic attacks (TIAs). The PERFORM Vascular Project will evaluate the effect of terutroban on progression of atherosclerosis, as assessed by change in carotid intima-media thickness (CIMT) in a subgroup of patients.
\end{abstract}

M. G. Hennerici

Department of Neurology, University of Heidelberg,

Mannheim, Germany

\section{L. Bots}

Julius Center for Health Sciences and Primary Care,

University Medical Center Utrecht,

Utrecht, The Netherlands

I. Ford

Robertson Centre for Biostatistics,

Glasgow, UK

S. Laurent

Department of Pharmacology and INSERM U970,

Hôpital Européen Georges Pompidou,

Paris, France

P. J. Touboul

Department of Neurology and Stroke Center, Hôpital Bichat,

Paris, France

M. G. Hennerici $(\square)$

University of Heidelberg, Universitätsmedizin Mannheim UMM,

Theodor-Kutzer-Ufer,

68135 Mannheim, Germany

e-mail: hennerici@neuro.ma.uni-heidelberg.de
Methods and results The Vascular Project includes structural (CIMT, carotid plaques) and functional (carotid stiffness) vascular studies in all patients showing at least one carotid plaque at entry. Expected mean follow-up is 36 months. Primary endpoint is rate of change of CIMT. Secondary endpoints include emergent plaques and assessment of carotid stiffness. 1,100 patients are required for $90 \%$ statistical power to detect treatment-related CIMT difference of $0.025 \mathrm{~mm}$. The first patient was randomized in April 2006.

Conclusions The PERFORM Vascular Project will investigate terutroban's effect on vascular structure and function in patients with a history of ischemic stroke or TIAs.

Key words Antiatherosclerotic activity · Antiplatelet agent - Atherosclerosis · Carotid intima-media thickness . Ischemic stroke $\cdot$ Carotid plaque $\cdot$ Clinical trial

\section{Introduction}

Terutroban is a specific thromboxane prostaglandin (TP) receptor antagonist [1]. It is an antiplatelet agent, at least as effective as aspirin [2] with potential additional antiatherosclerotic and antivasoconstrictive properties. It is expected to be useful in the secondary prevention of cerebrovascular and cardiovascular events in patients with a history of ischemic stroke and recent transient ischemic attacks (TIAs). This is currently being evaluated in a large-scale ongoing phase III randomized controlled trial, PERFORM (Prevention of 
cerebrovascular and cardiovascular Events of ischemic origin with teRutroban in patients with a history oF ischemic strOke or tRansient ischeMic attack). The PERFORM study includes 19,119 patients, who are randomly allocated to treatment with either terutroban $30 \mathrm{mg} /$ day or aspirin $100 \mathrm{mg} /$ day, and will be followed up for at least 3 years. The rationale and design of the PERFORM study have been described elsewhere, as well as the characteristics of the whole population at baseline [3, 4].

PERFORM is powered to detect the superiority of treatment with terutroban versus aspirin for prevention of the primary endpoint, which is a composite of fatal and nonfatal ischemic stroke, fatal and nonfatal myocardial infarction, or other vascular death. Here we describe the rationale and design of an ancillary study of PERFORM, the PERFORM Vascular Project, which has been specifically designed to study the effect of terutroban on the progression of atherosclerosis, as assessed by carotid intima-media thickness (CIMT), carotid plaques, and carotid stiffness in a subgroup of PERFORM participants.

\section{Rationale}

TP receptors are deeply involved in the atherosclerotic process whether they are located on platelets, immune cells (monocytes/macrophages or lymphocytes), or vascular cells (smooth muscle cells, endothelial cells). Indeed, injuryinduced vascular proliferation is reduced in mice that are genetically deficient in TP receptors. In contrast, this response is increased in mice that have been genetically modified and overexpress TP receptors (TPOE mice), but blocked when TPOE mice are treated with terutroban [5]. Inhibition of the TP receptors with an agent such as terutroban can therefore be expected to have a broad action on atherosclerosis. This had been shown for the first time in APOE deficient mice, in which terutroban, in contrast to aspirin, decreased atherosclerotic plaque formation most likely related to its anti-adhesive properties [6]. The antiatherosclerotic activity of terutroban has further been investigated in several animal models. In apobec-1/LDL receptor double knockout mice, terutroban delayed atherogenesis at the arch of the aorta compared with placebo and compared with a specific COX1/COX2 inhibition by indomethacin [7]. In cholesterol-fed New Zealand rabbits, an established animal model for advanced atherosclerosis, terutroban induced a regression of atherosclerotic plaque, as well as a significant reduction in the markers for macrophages, apoptotic cells, metalloproteinases, and endothelin1 , and a relative increase in vascular smooth muscle cells, suggesting that terutroban not only halts the progression of atherosclerosis, but also transforms lesions towards a more stable phenotype [8]. These results are in line with those from another animal studies, in which terutroban was found to enhance atherosclerotic lesion stability [9].

As regards terutroban's effects on vasoreactivity and endothelial function, this has been evaluated in atherosclerotic patients with endothelial dysfunction receiving treatment with aspirin. Terutroban improved endotheliumdependent dilatation evaluated on the forearm versus placebo. These results strongly support beneficial vascular effects of terutroban through a platelet-independent mechanism in patients already treated with aspirin [10].

Atherosclerosis is a dynamic and progressive inflammatory disease that precedes clinical vascular events such as myocardial infarction and ischemic stroke, and can be assessed non-invasively in a valid and reproducible manner with carotid ultrasound. In clinical trials, Carotid IntimaMedia Thickness (CIMT) is used as a surrogate endpoint for atherosclerosis. The association between CIMT and cardiovascular risk factors has been demonstrated, and CIMT, as measured by B-mode ultrasonography, has been found to be a powerful predictor of cardiovascular and cerebrovascular outcomes [11-15]. This non-invasive technique allows sequential follow-up measurements in epidemiological and clinical trials with the best precision when using standardized methods [16]. Moreover, vascular ultrasound can also be used to assess atherosclerotic changes in the arterial wall expressed by the occurrence of carotid plaque [17] and by local arterial stiffness. In the PERFORM Vascular Project, CIMT will be evaluated over 3 years to allow for sufficient time to detect antiproliferative and antiatherogenic activity. This is in line with the study duration of other trials that investigated the effects of various agents on CIMT [18-21].

\section{Design}

The PERFORM Vascular Project is an ancillary study of the randomized, double-blind, controlled, parallel-group, phase III PERFORM study, which is ongoing in 802 centers in 46 countries [3, 4]. Of these, 52 centers in 16 countries have agreed to participate in the Vascular Project.

The main PERFORM study included patients aged $\geq 55$ years, who had a cerebral or retinal infarction in the last 3 months preceding inclusion or TIA in the last 8 days, and who were stable at inclusion with no intracranial hemorrhage or non-ischemic neurological disease. The inclusion criteria for the PERFORM Vascular Project were men or women participating in the main study aged between 55 and 80 years, with validated baseline (M0) vascular evaluation including (1) an assessment of the quality of the images (positioning, recording); (2) confirmation that both 
common carotid arteries (CCA) were visible and at least one carotid plaque was present; and (3) at least one CIMT measurement on a CCA segment free of plaque was possible. Patients were excluded from the Vascular Project because of absence of carotid plaque, carotid stenosis $\geq 70 \%$ or complete carotid obstruction (CCA, internal carotid artery [ICA], or bifurcation), or a history of carotid surgery, including percutaneous intervention (stent placement).

The trial profile of the PERFORM Vascular Project is shown in Fig. 1. The first (baseline) ultrasonographic assessment of vascular function (CIMT) is performed at randomization (i.e., the $\mathrm{M} 0$ visit) prior to treatment allocation. Ultrasonographic vascular explorations will then be performed within 15 days of the M12 (12 months after baseline), M24, M36 (if applicable) visits, and then at the final visit, according to a prespecified procedure.

Participants in the Vascular Project were fully informed and written consent was obtained according to local regulatory requirements.

\section{Assessments}

Collection of ultrasound data and subsequent analysis of the ultrasound images is being carried out in close agreement with the Mannheim Carotid Intima-Media Thickness Consensus [16].

\section{Ultrasonographic acquisition}

B-mode scanning is being performed by sonographers trained and certified specifically for the PERFORM Vascular Project. CIMT measurements are made using Bmode duplex ultrasound scanner (linear array $\geq 7 \mathrm{MHz}$, or broadband $5-10 \mathrm{MHz}$ or higher). Vascular centers have been provided with a medical DICOM recorder (MDR) in order to transfer carotid B-Mode ultrasound images in a uniform DICOM format to the centralized imaging core laboratory (Bioclinica). The image depth will be standardized at $4 \mathrm{~cm}$ or close to that, with a single focus zone and no zoom, a high frame rate, low persistence, and optimized image gain settings. The region of interest in the CCA will be imaged at baseline at an angle that displays the clearest near and far wall boundaries (optimal angle) and at three predetermined angles of $30^{\circ}$ increments according to the Meijer carotid arc. The optimal angle at baseline is noted, and will be consulted on subsequent visits. A quality control procedure regarding image quality, protocol compliance, and completeness of study data is being performed for each vascular examination.

\section{Ultrasonographic analysis}

For the measurement of CIMT, the region of interest (ROI) is defined as the CCA segment at least $10 \mathrm{~mm}$ below the bifurcation (Fig. 2). CIMT will be assessed in longitudinal view on the far wall of right and left CCA on a $10-\mathrm{mm}$ length in a region free of plaque. For assessment of plaque, longitudinal and transversal images will be recorded in the right and left carotid trees (CCA, bifurcation, and ICA) from the origin of the cervical CCA to the first $30 \mathrm{~mm}$ of the ICA. Image analysis will be performed using the Arterial Measurement System II and automated contour detection after positioning a measurement box $(10 \mathrm{~mm}$ wide) will be verified and manually corrected if needed [22]. CIMT assessment will be performed by batch reading after completion of all vascular examinations for each patient.

If a plaque is present, ultrasound images will be recorded, as well as will information on the number of plaques present and their location. A plaque is defined as a focal structure that encroaches into the arterial lumen of at least $0.5 \mathrm{~mm}$ or $50 \%$ of the surrounding CIMT value or demonstrates a thickness of $\geq 1.5 \mathrm{~mm}$ as measured from the media-adventitia interface to the intima-lumen interface [16]. For qualitative assessment, plaques will be graded as follows: (1) none (complete absence, CIMT thickening may be observed); (2) minimal (small isolated thickening); (3) moderate (visible plaque, causing a modest degree diameter reduction); (4) severe (significant plaque, visualized on
Fig. 1 The PERFORM Vascular Project: Study design. TIA, transient ischemic attack.

* Prior to first study drug intake

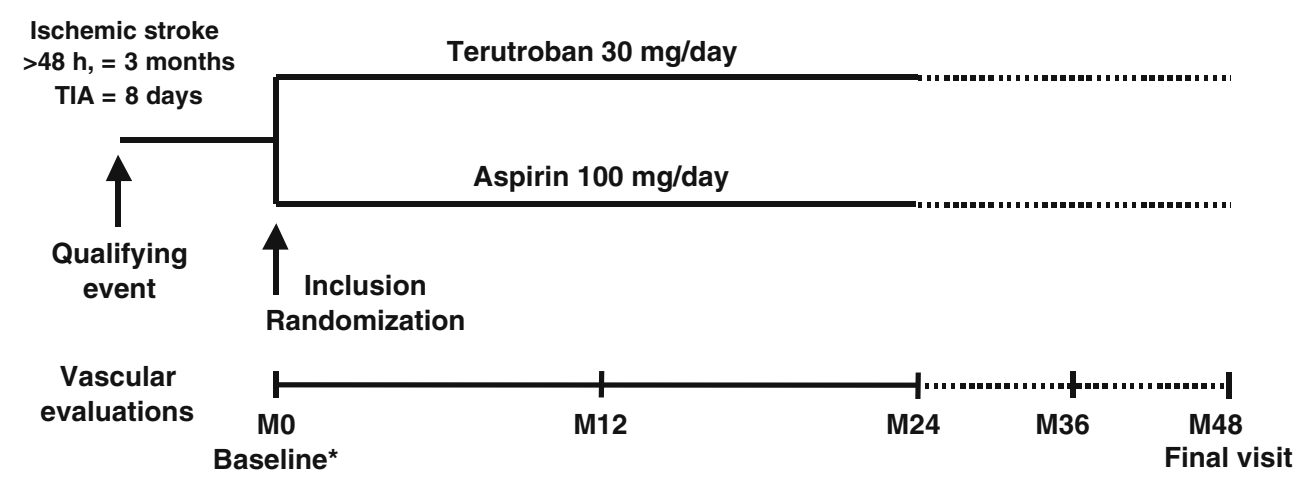




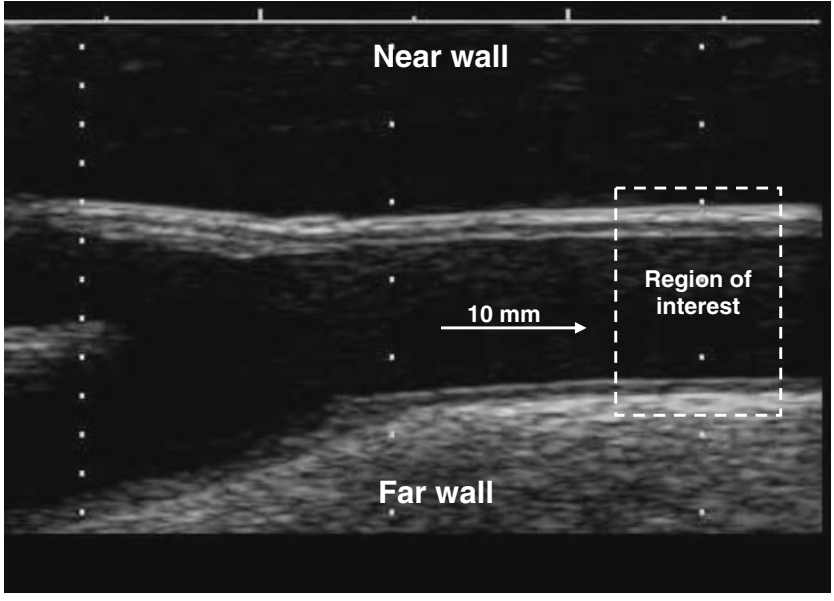

Fig. 2 Carotid tree: region of interest. Left: internal and external carotid arteries; Middle: carotid bifurcation; Right: common carotid artery

several different scan projections, causing clear reduction in diameter); or (5) occlusion [17, 23].

Carotid stiffness will be assessed, as previously described, using ultrasound and blood pressure [24]. Stroke changes in carotid diameter will be calculated from the 5-second video clip acquired at the optimal angle from automated boundary tracing during at least three cardiac cycles. Brachial blood pressure recordings made after completion of the left and right carotid CIMT measurements will be used. Central pulse pressure will be calibrated from the diameter-time curve and brachial blood pressure [25]. Distensibility is defined as the ratio of relative stroke change in diameter on pulse pressure. Carotid stiffness is expressed as the inverse of the square root of distensibility, to give similar information to aortic stiffness [26].

All ultrasound images will be evaluated and analyzed centrally by an independent imaging core laboratory (Bioclinica, Leiden, The Netherlands, BI). The core laboratory will perform a centralized quality control for ultrasound acquisition, notably for image quality and protocol deviations, and variability. Assessment of local arterial stiffness will be evaluated and analyzed centrally at the Hôpital Georges Pompidou Core Centre (HEGP Paris, France), from ultrasound images provided by Bioclinica. Cineloops will be analyzed by Bioclinica and diameter values will be extracted along time. Time series will be sent to HEGP and analyzed with dedicated software.

Quality control and reproducibility

The quality control process included a uniform training program for the sonographers and variability studies for the central readers. An initial reader variability study has been performed for two readers on a random selection of 58 independent images from baseline and M12 scans. The inter-reader Intraclass correlation coefficient for the mean common CIMT was 0.94 (95\% confidence interval [CI]: $[0.872 ; 0.963])$. Intra-reader intraclass correlation coefficients for the mean common CIMT were 0.93 for reader 1 (95\% CI: [0.893; 0.961]) and 0.92 for reader 2 (95\% CI: $[0.875 ; 0.954])$. Two additional variability assessments will be performed, one before the beginning of the batch reading to certify the central readers, and another during the batch reading. Should the reader intraclass correlation coefficient be less than 0.80 , a new training session will be held, and the reader will complete a new assessment of reproducibility.

As regards plaque reporting, studies of reproducibility between the core laboratory and the sonographers have been carried out. Plaque score and count in 47 subjects with an available M0 and M12 scan were analyzed by the core laboratory and compared with the sonographers' reports. The increase in the plaque count was evaluated at $+14 \%$ by the core laboratory between M12 and M0 compared with $+13 \%$ by the sonographers. Similar results were found in a second study among 38 patients with M0, M12, and M24 available timepoints. These findings comply with those reported by other groups [23].

\section{Endpoints}

The primary endpoint of the PERFORM Vascular Project is the annualized rate of change in the mean common CIMT ( $\mathrm{mm}$ per year), on the basis of the assessments made at baseline (M0), M12, M24, M36 (if applicable) and/or at the final visit. The secondary endpoints are the change in mean common CIMT from baseline (M0) to final visit (mm), the number of emergent carotid plaques in both carotid systems (left and right) and the change in carotid stiffness from baseline to final visits.

\section{Baseline characteristics}

Recruitment into the PERFORM Vascular Project started in February 2006 and the last patient was randomized in March 2008. A total of 1,141 patients were randomized from 52 vascular centers in 16 countries; $37 \%$ of the population were recruited in Western Europe, 58\% in Eastern Europe, 3\% in Australia, and 2\% in Canada. Demographic characteristics and past medical history are presented in Table 1.

The mean age of the population was $66.2 \pm 6.5$ years (range 54-80 years), with $12 \%$ of patients over 75 years. Sixty-six percent of the population was male. The most 
Table 1 Baseline characteristics of the PERFORM Vascular Project population

\begin{tabular}{ll}
\hline Total patients & 1,141 \\
Male, \% & 66 \\
Mean age, years, mean \pm SD & $66.2 \pm 6.5$ \\
Age, \% & \\
$<65$ years & 44 \\
$\geq 65$ to $<75$ years & 44 \\
$\geq 75$ years & 12 \\
Ethnicity, \% & \\
Caucasian & 99 \\
Asian & 0.3 \\
Black & 0.1 \\
Other & 0.1 \\
Smoking \% & \\
Never smoked & 44 \\
Current smoker & 28 \\
Stopped smoking $>6$ months & 28 \\
Medical history, \% & \\
Hypertension & 86 \\
Hypercholesterolemia & 54 \\
Diabetes & 29 \\
Prior history of cerebral infarction & \\
Angina pectoris & 12 \\
Hypertriglyceridemia & 10 \\
Prior history of TIA & \\
Myocardial infarction & 62 \\
Peripheral artery disease & \\
Previous treatments, ${ }^{a} \%$ & \\
Statins & \\
Angiotensin-converting enzyme inhibitors & \\
\hline
\end{tabular}

${ }^{a}$ Recorded between index stroke and randomization

frequent risk factors were hypertension (86\%), hypercholesterolemia (54\%), and diabetes (29\%). Twelve percent of the population reported a previous ischemic stroke and $8 \%$ a previous TIA, while $8 \%$ had a history of myocardial infarction. These data are largely comparable to the main study population characteristics [4], except for the geographic distribution of the recruitment, which resulted in a higher proportion of patients of Caucasian origin in the Vascular Project (99\% versus $83 \%$ in the main study) and a higher proportion of patients with hypercholesterolemia ( $54 \%$ versus $48 \%$ in the main study). This is most likely due to the inclusion criteria of patients in the Vascular Project, which require patients with at least one carotid plaque at the baseline examination. In addition, the Vascular Project population is slightly more likely to be receiving angiotensin-converting enzyme inhibitor than the main study population (63\% versus $55 \%)$.

\section{Data analytic approach}

The PERFORM Vascular Project results will be analyzed by the Robertson Centre for Biostatistics, University of Glasgow, UK, and subsequently validated by the Biometry Division of the Institut de Recherches Internationales Servier. The expected mean follow-up is 36 months. Assuming an expected standard deviation of $0.11 \mathrm{~mm}$ of the rate of change in common CIMT and a treatment withdrawal rate of $10 \%$ per year, we estimate that 1,100 patients are necessary to detect a treatment-related CIMT change of $0.025 \mathrm{~mm}$ (i.e., $0.01 \mathrm{~mm} /$ year) for a statistical power of at least $90 \%$ using a two-sided test with a $5 \%$ type 1 error rate.

The analyses will be performed in all patients in the full analysis set and in the per protocol set, i.e. patients from the full analysis set who are compliant with the protocol. The type 1 error will be set at 5\% (two-sided) for all statistical tests and $95 \%$ CIs will be provided.

The effect of terutroban versus aspirin on the primary endpoint (rate of change of common CIMT) will be analyzed using a linear mixed-effects model adjusted for baseline value. Comparison of the treatment groups for the secondary endpoints of mean common CIMT and carotid stiffness will be performed in a similar manner using a general linear model with baseline value as a covariate. The number of emergent carotid plaques will be compared for the two groups by Poisson regression.

\section{Organization}

The organization of the main PERFORM study has been described at length elsewhere [3]. The PERFORM Vascular Project is supervised by the Vascular Scientific Committee, comprising five members: Prof M.G. Hennerici (Chairman), Prof I. Ford, Prof M.L. Bots, Prof S. Laurent, and Prof P.J. Touboul. Independent centralized reading of the ultrasound images is performed by Bioclinica, Leiden, The Netherlands. The investigators of the PERFORM Vascular Project are listed at the end of this article.

\section{Conclusion}

The PERFORM Vascular Project is part of the largest ongoing trial in secondary prevention of stroke and will study prospectively the effect of terutroban as compared to aspirin on the progression of the CIMT in a large number of patients.

Acknowledgments Servier is the sponsor of the PERFORM study. We would like to thank all the participants in the ultrasound acquisition, and Rudy Meijer and Bioclinica for their contribution. 
Conflict of interest statement The authors have all received honoraria, research grants, or both from Servier.

PERFORM Vascular Project Investigators Australia, C. Anderson, G. Donnan, T. Phan; Belgium, V. Thijs; Canada, D. Selchen, D. Spence; Czech Republic, M. Bar, P. Kanovsky, I. Rektor, O. Skoda, D. Vaclavik; France, C. Lucas, T. Rosolacci, P. Trouillas; Germany, B. Griewing, M. Hennerici, A. Hetzel, M. Kaps, W. Koehler, J. Roether; Hungary, A. Csanyi, L. Csiba, Z. Kaposzta, J. Nikl, G. Panczel, E. Pongracz, N. Szegedi, A. Valikovics; Italy, C. Cappelletti, V. Di Piero, P. Prati, P.M. Rossini; The Netherlands, M.L. Bots, L.J. Kappelle; Poland, A. Czlonkowska, W. Kozubski, A. Kuczynska-Zardzewialy, H. Kwiecinsky; Portugal, A.V. Salgado; Russia, D. Butko, E. Gusev, P. Kamchatnov, S. Kotov, V. Parfenov, V. Skvortsova, L. Stakhovskaya; Slovenia, B. Zvan; Spain, J. Alvarez Sabin, J. Castillo Sanchez, D. Jimenez Martinez, J. Serena Leal, J.M. Trejo Gabriel, J. Vivancos Mora; Switzerland, R.W. Baumgartner, L. Hirt; United Kingdom, K. Lees, R. MacWalter.

Open Access This article is distributed under the terms of the Creative Commons Attribution Noncommercial License which permits any noncommercial use, distribution, and reproduction in any medium, provided the original author(s) and source are credited.

\section{References}

1. Sorbera LA, Serradell N, Bolos J, Bayes M. Terutroban sodium. Prostanoid tp receptor antagonist, antithrombotic agent, antiatherosclerotic agent. Drugs Future. 2006;31:867-73.

2. Fiessinger JN. S18886, a new specific TP-receptor antagonist, is safe and as effective as aspirin in inhibiting platelet aggregation in patients with peripheral arterial disease. Eur Heart J. 2004;25:573.

3. Bousser MG, Amarenco P, Chamorro A, et al. Rationale and design of a randomized, double-blind, parallel-group study of terutroban $30 \mathrm{mg} /$ day versus aspirin $100 \mathrm{mg}$ /day in stroke patients: the Prevention of cerebrovascular and cardiovascular Events of ischemic origin with teRutroban in patients with a history oF ischemic strOke or tRansient ischeMic attack (PERFORM) study. Cerebrovasc Dis. 2009;27:509-18.

4. Bousser MG, Amarenco P, Chamorro A, et al. The Prevention of cerebrovascular and cardiovascular Events of ischemic origin with teRutroban in patients with a history oF ischemic strOke or tRansient ischeMic attack (PERFORM) study: baseline characteristics of the population. Cerebrovasc Dis. 2009;27:608-13.

5. Cheng Y, Austin SC, Rocca B, et al. Role of prostacyclin in the cardiovascular response to thromboxane A2. Science. 2002;296:539-41.

6. Cayatte AJ, Du Y, Oliver-Krasinski J, Lavielle G, Verbeuren TJ, Cohen RA. The thromboxane receptor antagonist S18886 but not aspirin inhibits atherogenesis in apo E-deficient mice: evidence that eicosanoids other than thromboxane contribute to atherosclerosis. Arterioscler Thromb Vasc Biol. 2000;20:1724-8.

7. Egan KM, Wang M, Fries S, et al. Cyclooxygenases, thromboxane, and atherosclerosis: plaque destabilization by cyclooxygenase-2 inhibition combined with thromboxane receptor antagonism. Circulation. 2005; 111:334-42.

8. Viles Gonzalez JF, Fuster V, Corti R, et al. Atherosclerosis regression and TP receptor inhibition: effect of S18886 on plaque size and composition - a magnetic resonance imaging study. Eur Heart J. 2005;26:1557-61.

9. Worth NF, Berry CL, Thomas AC, Campbell JH. S18886, a selective TP receptor antagonist, inhibits development of atherosclerosis in rabbits. Atherosclerosis. 2005;183:65-73.
10. Belhassen L, Pelle G, Dubois Rande JL, Adnot S. Improved endothelial function by the thromboxane A2 receptor antagonist S18886 in patients with coronary artery disease treated with aspirin. J Am Coll Cardiol. 2003;41:1198-204.

11. O’Leary DH, Polak JF, Kronmal RA, Manolio TA, Burke GL, Wolfson Jr SK. Carotid-artery intima and media thickness as a risk factor for myocardial infarction and stroke in older adults. Cardiovascular Health Study Collaborative Research Group. N Engl J Med. 1999;340:14-22.

12. Bots ML, Hoes AW, Koudstaal PJ, Hofman A, Grobbee DE. Common carotid intima-media thickness and risk of stroke and myocardial infarction: the Rotterdam Study. Circulation. 1997;96: $1432-7$.

13. Chambless LE, Heiss G, Folsom AR, et al. Association of coronary heart disease incidence with carotid arterial wall thickness and major risk factors: the Atherosclerosis Risk in Communities (ARIC) Study, 1987-1993. Am J Epidemiol. 1997;146:483-94.

14. Chambless LE, Folsom AR, Clegg LX, et al. Carotid wall thickness is predictive of incident clinical stroke: the Atherosclerosis Risk in Communities (ARIC) study. Am J Epidemiol. 2000;151:478-87.

15. Lorenz MW, Markus HS, Bots ML, Rosvall M, Sitzer M. Prediction of clinical cardiovascular events with carotid intimamedia thickness: a systematic review and meta-analysis. Circulation. 2007;115:459-67.

16. Touboul PJ, Hennerici MG, Meairs S, et al. Mannheim carotid intima-media thickness consensus (2004-2006). An update on behalf of the Advisory Board of the 3rd and 4th Watching the Risk Symposium, 13th and 15th European Stroke Conferences, Mannheim, Germany, 2004, and Brussels, Belgium, 2006. Cerebrovasc Dis. 2007;23:75-80.

17. Zureik M, Ducimetiere P, Touboul PJ, et al. Common carotid intima-media thickness predicts occurrence of carotid atherosclerotic plaques: longitudinal results from the Aging Vascular Study (EVA) study. Arterioscler Thromb Vasc Biol. 2000;20:1622-9.

18. Amarenco P, Labreuche J, Lavallee P, Touboul PJ. Statins in stroke prevention and carotid atherosclerosis: systematic review and up-to-date meta-analysis. Stroke. 2004;35:2902-9.

19. Kodama M, Yamasaki Y, Sakamoto K, et al. Antiplatelet drugs attenuate progression of carotid intima-media thickness in subjects with type 2 diabetes. Thromb Res. 2000;97:239-45.

20. Pitt B, Byington RP, Furberg CD, et al. Effect of amlodipine on the progression of atherosclerosis and the occurrence of clinical events. PREVENT Investigators. Circulation. 2000;102:1503-10.

21. Crouse III JR, Raichlen JS, Riley WA, et al. Effect of rosuvastatin on progression of carotid intima-media thickness in low-risk individuals with subclinical atherosclerosis: the METEOR Trial. JAMA. 2007;297:1344-53.

22. Liang Q, Wendelhag I, Wikstrand J, Gustavsson T. A multiscale dynamic programming procedure for boundary detection in ultrasonic artery images. IEEE Trans Med Imaging. 2000;19: $127-42$.

23. Dogan S, Meijer R, Grobbee DE, et al. Assessment of carotid plaque progression with ultrasound in a low-risk population. Presented at the International Symposium on Atherosclerosis, Boston, Mass, USA. 2009.

24. Paini A, Boutouyrie P, Calvet D, Tropeano AI, Laloux B, Laurent S. Carotid and aortic stiffness: determinants of discrepancies. Hypertension. 2006;47:371-6.

25. Van Bortel LM, Balkestein EJ, van der Heijden-Spek JJ, et al. Non-invasive assessment of local arterial pulse pressure: comparison of applanation tonometry and echo-tracking. J Hypertens. 2001;19:1037-44.

26. Laurent S, Cockcroft J, Van BL, et al. Expert consensus document on arterial stiffness: methodological issues and clinical applications. Eur Heart J. 2006;27:2588-605. 